\title{
Integrating promotive, preventive, and curative health care services at hospitals and health centers in Addis Ababa, Ethiopia
}

This article was published in the following Dove Medical Press journal: Journal of Multidisciplinary Healthcare

\section{Netsanet Fetene \\ Wendimagegn' \\ Marthie C Bezuidenhout ${ }^{2}$ \\ 'Health Management and Leadership, Yale Global Health Leadership Institute (GHLI), Addis Ababa, Ethiopia; ${ }^{2}$ Department of Health Studies, University of South Africa, Pretoria, South Africa}

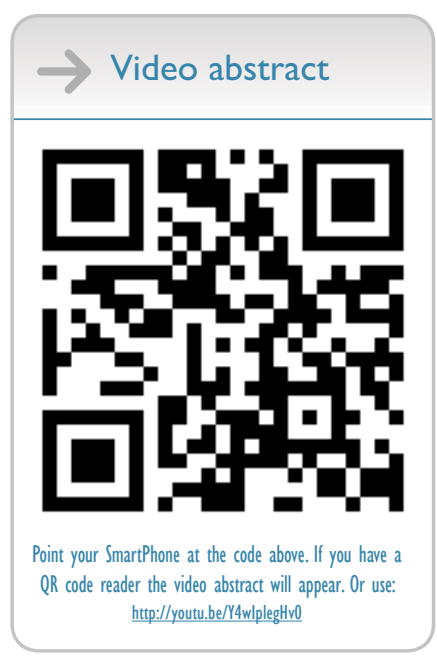

Correspondence: Netsanet Fetene Wendimagegn

Yale Global Health Leadership Institute (GHLI), PO Box 5874, Addis Ababa, Ethiopia

Tel +251910137506

Email netsanetfetene@gmail.com
Background: The current trend in patients' disease management is mostly aimed at addressing their present health complaints; the focus is thus purely curative. As the limits of curative medicine become apparent and the cost of medical care escalates, disease prevention is gaining prominence. Factors that contribute to unreliable delivery of an integrated health care service are worth investigation. This study explores the extent to which health promotion and disease prevention services are integrated to curative health care and identifies the factors associated with not reliably providing the services.

Methods: A cross-sectional quantitative study using an exploratory and descriptive design was used to explore and describe the extent of health promotion, preventive, and curative health care services provision, and investigated factors related to low performance. Phase I of the study examined the degree of promotive and preventive health care provision at hospitals and health centers while investigating the staffing and equipment and supply status of the facilities. Phase II, using the Delphi consensus-seeking process, focused on the validation of the findings from Phase I.

Results: Of all patients who attended health facilities, only $2.4 \%(n=20)$ received optimal health promotion services. Disease prevention services were optimally provided to only $3.6 \%$ $(n=30)$ patients. Integrated health promotion and disease prevention services were provided to only $0.8 \%(n=7)$ patients. The main reasons for not providing an integrated health care service were shortage of skilled health staff, equipment, medication, protocols, and guidelines, and high service cost, poor patient awareness, and health professionals' focus on curative health care. Conclusion: Health service providers were not routinely conducting patient-specific health promotion, disease prevention, and integrated health care services, losing the opportunities of patient's presence for health promotion and diseases prevention purposes. Addressing barriers can help with integrating health promotion and disease prevention services to the curative health care services.

Keywords: integrated health service, disease prevention, health promotion, curative care, non-communicable diseases

\section{Background}

Presently, as the limits of curative medicine become apparent and the cost of medical care escalates in all countries, disease prevention is gaining prominence. ${ }^{1}$ While health promotion and disease prevention can be distinguished conceptually, they can hardly be distinguished in practice. Most general measures do both at the same time. ${ }^{2}$ Health promotion programs can be integrated into preventive services in health care systems. ${ }^{3}$ Health promotion is any combination of educational, organizational, economic, and environmental support systems with the aim of ensuring behavior and conditions of living conducive to health. ${ }^{4}$ Health promotion is not just the responsibility of the health 
sector, but goes beyond healthy lifestyles and well-being. ${ }^{5}$ Prevention in health care refers to action that prevents disease occurrence that includes actions aimed at eradicating, eliminating, or minimizing the impact of disease and disability, or if none of these is feasible, retarding the progress of disease and disability. ${ }^{6}$ Preventive medicine encompasses both the care of individual patients and public health practices that focus on the prevention of disease rather than treatment per se. ${ }^{7}$ Integration of health promotion and disease prevention involves strategies that target several risk factors, use multiple strategies at various levels of influence, and require inter-sectorial action. Integration entails multiplicity ( $>1$ risk factor, level, sector, agent), and synergy resulting from multiplicity. ${ }^{6}$

During patients' clinical evaluation, the "need to routinely ask patients (and when appropriate family members) what matters most to them in the experience of illness and treatment tends to be left out of the interaction". ${ }^{8}$ All clinic contacts, whether for acute, chronic, or preventive reasons, are valuable opportunities for health promotion and disease prevention activities to take place. ${ }^{9}$ Moreover, several of the services most likely to go undelivered represent substantial opportunities not only to decrease the clinically preventable burden of disease but also to improve the cost-effectiveness of care delivered. ${ }^{10}$ This is particularly important for most chronic diseases.

According to the $\mathrm{WHO},{ }^{11}$ the effect of non-communicable diseases (NCDs) is leading to a global crisis and requires a concerted global response. NCDs undermine social and economic development throughout the world, threaten the achievement of internationally agreed upon development goals, and increase inequalities between countries and populations. This is because two of three deaths each year are attributable to NCDs. NCDs often cause slow and painful deaths after prolonged periods of disability, in all regions of the world, and total numbers of NCD deaths are rising and the double burden of disease places enormous strains on resource-deficient health systems. NCDs also disproportionately affect individuals who are poor, thus increasing inequalities. ${ }^{12}$ This situation is especially tragic considering that at least $80 \%$ of all heart diseases, stroke, and diabetes conditions are preventable. ${ }^{13}$ Despite the threat to human development, and the availability of affordable, cost-effective, and feasible interventions, most countries, development agencies, and foundations neglect the NCD crisis. ${ }^{12}$

In low- to middle-income countries, the proportion of premature NCD deaths under 60 years rose to $28 \%$, more than double the proportion in high-income countries. Some risk factors including tobacco use among men, overweight, and obesity have become more common in middle-income countries. ${ }^{14}$ In Ethiopia, NCDs account for an estimated 34\% of all deaths. ${ }^{15}$ The Ethiopia Ministry of Health acknowledged the evolving double burden of disease types presenting as a mix of persistent, emerging, and re-emerging infectious diseases and increasing NCDs and injuries. ${ }^{16}$

In 2014, Misganawa et al found a high prevalence of risk factors for NCDs, including tobacco use, harmful use of alcohol, hypertension, overweight/obesity, high glucose levels, and khat chewing, mainly in the urban population aged $\geq 15$ years. ${ }^{17}$ Khat is an indigenous plant in Ethiopia, Kenya, and Yemen and chewing khat is linked to hypertension and oral cancer. ${ }^{18}$ In a study of 3,713 adults in Addis Ababa, Tesfaye et al ${ }^{19}$ found that high blood pressure (BP) was widely prevalent $(31.5 \%$ of the males and $28.9 \%$ of the females); overweight ( $20 \%$ of the males and $38 \%$ of the females); and physical inactivity (17\% of the males and $31 \%$ of the females) were important determinants of high BP, and $25 \%$ had a sedentary lifestyle. They referred this change as "a silent epidemic". ${ }^{19}$

In Ethiopia, like many low- and middle-income countries, the current trend in patients' disease management in public hospitals and health centers is almost entirely aimed at addressing only their present health complaints; the focus is thus purely curative. In 2014, a household health service utilization study indicated that only $0.63 \%$ of out-patient health service users received medical check-ups or preventive health care. ${ }^{20}$ The end result of failing to provide an integrated promotive, preventive, and curative health care management approach continues to pose a risk of ill health in patients and increased disease burden to the country. ${ }^{21}$ However, patients can greatly benefit by preventing illness and optimizing their health by adopting healthy lifestyles if they are appropriately informed, taught, and guided through health education. ${ }^{3}$ Therefore, both symptomatic and asymptomatic patients seeking health care should be assessed for the need of health promotion and preventive services during routine clinic health care visits (Institute for Clinical Systems Improvement). ${ }^{9}$ A double burden of disease, the co-occurrence of infectious and chronic NCDs, requires integrated control strategies that should begin in the primary health care system with maximum involvement of all specialized personnel. ${ }^{22}$

Not reliably delivering an integrated health care service could be caused by several factors. There are patient and health service provider-based reasons for failure to deliver health promotion and preventive services. The health promotion and preventive services received by patients partly depend on the type of health care professionals, their knowledge and commitment to these services, and the health care facility's readiness to provide promotive and preventive ser- 
vices. ${ }^{23}$ According to the WHO, in low-income countries, the level of integrating NCD prevention and curative health care at primary health care level mainly depends on the capacity of the countries in relation to funding; staffing; availability of a unit responsible to provide prevention services; presence of policy, plan, or strategy; approved evidence-based national guidelines; and protocols or standards for managing selected preventable diseases. ${ }^{24}$ In addition, health care organizations may lack incorporating and implementing new systems of preventative health care that include decision support tools, preferably integrated into the medical record, mechanisms that generate alerts and reminders when services are due. ${ }^{25}$

Understanding the level of health promotion and disease promotion to the curative health care and identifying the barriers for integration motivated the researcher to conduct the study. The aim of the study was to explore the extent to which health promotion and disease prevention services are integrated to curative health care at public general hospitals and health centers and identify the factors associated for not reliably providing the services at the health facilities.

\section{Methods}

A cross-sectional quantitative study approach using an exploratory and descriptive design was used to explore and describe the current situation of health promotion, preventive, and curative health care services. The study explored the extent of health promotion and disease prevention service provision and investigated factors related to low performance in this regard. The study was conducted in two phases. Phase I examined the degree of promotive and preventive health care provision at the hospitals and health centers while investigating the staffing and equipment status of the facilities. Phase II, using the Delphi consensus-seeking process, focused on validation of the findings from Phase I and developing an integrated health service (IHS) framework for the improvement of health promotion and disease prevention services at hospitals and health centers. This study focuses on the findings from Phase I.

The study was conducted in Addis Ababa, the capital of Ethiopia. It focused on public hospitals and health centers in Addis Ababa. The government health care facilities in Addis Ababa are classified as general hospitals, specialized referral hospitals, and health centers. During the study period, the Ethiopia Ministry of Health Policy and Planning Directorate reported that there were 47 public hospitals and health centers registered in Addis Ababa. ${ }^{26}$ From the total site sample frame, $50 \%$ were taken proportionately as sample sites (three government hospitals, 19 government health centers) to par- ticipate in the study. In the 22 selected health services, the medical inpatient department (IPD) and outpatient department (OPD) sections were targeted for data collection. The data were collected from the patients by means of structured interviews and from the health service managers by means of self-administered questionnaires. In the second phase, questionnaires were distributed to the experts through the Delphi technique as a means of reaching consensus on findings from Phase I. Ten data collectors, registered nurses, and two supervisors, public health experts, along with the principal investigator, not affiliated to the hospital and clinics, participated in data collection process.

In the first phase of the study, the respondent population consisted of two strata, namely patients and health service managers. To be included in the study, the patients had to attend medical OPDs or IPDs of the 22 health facilities in the first stratum. Patients discharged from hospitals or health centers after having been admitted for at least 24 hours, and who were between 18 and 65 years of age and in stable condition, participated in the study. Since the proportion of patients who received optimal health promotion or disease prevention service was not known, 50\% proportion was taken to determine the maximum sample size. Considering the $95 \%$ CI, with the standard error of $5 \%, \mathrm{z}$ value at $95 \% \mathrm{CI}$ of 1.96 , the sample size calculated to be 385 . Allowance needs to be made for non-responders so that the additional number can be added on to the required sample size. ${ }^{27}$ Considering $10 \%$ non-response rate, the sample size for patient interview was made to be 424 . Since multistage sampling technique was used, the design effect (deff) should be applied. Design effect is not known before a survey is undertaken and can only be estimated afterward. A default value of 1.5-2.0 for deff is typically used by researchers in the formula for calculating the sample size. ${ }^{28}$ In order to obtain the maximum sample size in this study, a design effect of 2 was applied, thus making the total sample size for patient exit interviews as 848 . Some of the questions in the patient exit interview questionnaire were partly derived and modified from the Behavioral Risk Factor Surveillance System Questionnaire (BRFSS) prepared on health promotion and disease prevention developed by the Centers of Disease Control and Prevention. The BRFSS is a system of health-related telephone surveys that is used annually to collect state data about US residents regarding their health-related risk behaviors, chronic health conditions, and use of preventive services. ${ }^{29}$ Only questions from the BRFSS that could be applied to the local context and could be modified for the purposes of an exit interview were customized and used. 
The health service managers working in 22 health facilities formed the second stratum of the respondent population for the first phase of the study. The health service managers were the managers of the health facilities and comprised medical doctors, public health experts, and nurses. The data collected from the management group and patients during Phase I were analyzed with the assistance of a statistician using the SPSS version 21.0 program. Descriptive and inferential statistical techniques were used to analyze the collected data. Frequency tables, cross-tabulation, and Spearman's rho technique to compute the correlation were applied for comparison and association of results among dependent and independent variables.

In order to quantify the provision of health promotion and disease prevention services provided by health facilities, the following operational definitions were applied considering an expected integrated and comprehensive health care relevant to the patients' age and sex.

\section{Health promotion service provision}

In order to assess whether the health promotion activities were provided by health service providers working in hospitals and health centers, the study operationalized the provision of health promotion services to patients based on five selected health promotion activities. The selected health promotion activities were providing counseling on nutrition; checking patients on physical exercise routine and advice on its use; examining and counseling patients on how to prevent sexually transmitted infections (STIs); checking patients' housing and/ or environmental conditions; and offering written materials on health promotion to patients. Patients were categorized as receiving "optimal health promotion" services if they received $>4$ of the services; "sub-optimal" if they received one to three of the services, and "not received health promotion service" if none of the five selected services were provided.

\section{Disease prevention service provision}

Selected preventive activities for common non-communicable chronic diseases were used to measure disease prevention service provision by the hospitals and health centers. The chronic disease risk factors expected to be performed by health service providers in their preventive screening activities included BP monitoring, screening for cancer risks, screening for diabetes risks, checking on cigarette smoking and counseling, assessment for patient alcohol abuse, monitoring increased cholesterol, and assessing risks for HIV infection.
Of the seven preventive services, patients receiving four or more types of services were considered to receive "optimal" disease prevention service; receiving one to three services was considered "sub-optimal" preventive care; and receiving no preventive services was considered "not provided" with disease prevention services.

\section{IHS provision}

IHS provision by health facilities was considered when at least the minimum recommended list of both health promotion and disease prevention services was provided to the patients. Integrated services were considered to have been provided "optimally" if the sum of the promotive and preventive services was $\geq 7$ while if the sum of services was between 2 and 6, it was considered "sub-optimal". If no or only one type of health promotion or disease prevention service was provided, it was considered "no integrated service was provided."

When one or more of the variables are measured on an ordinal (ranking) scale, the appropriate correlation coefficient is Spearman's rank-order correlation coefficient. ${ }^{30}$ Health promotion, disease prevention, and integrated service provision were all ranked as outcomes of "not provided," "sub-optimal," and "optimally provide" based on the service provided to the respondents. In order to assess the relationship of these variables with the biographical, health facility-type and patient-related characteristics, the researchers applied the Spearman's rho technique to compute the correlation. The strength of correlation was measured as a correlation coefficient and symbolized as $r$.

In the second phase, 20 experts who were working in Ethiopia and had a minimum work experience of 10 years in the field of health promotion and disease prevention were selected to participate in the two rounds of Delphi consensus-seeking exercise. This phase, using the Delphi consensus-seeking process facilitated by the principal investigator, focused on validation of the finding from Phase I and developed an IHS framework for the improvement of health promotion and disease prevention services at hospitals and health centers. The Delphi technique is well suited as a method for consensus-building by using a series of questionnaires and multiple iterations to collect data from a panel of selected subjects. ${ }^{31}$ The content of the Delphi questionnaires was developed based on the findings of Phase 1 of this study, and the response alternatives were tied to a 5-point Likert scale, seeking the respondents' measure of agreement with the questions. Follow-up questions encouraged experts to 
provide comments in order to seek their views, recommendations, and suggestions on the issues listed. Agreement was considered to be reached when $75 \%$ of the experts agreed on an issue. ${ }^{32}$ Thereafter, further clarification and consensus were sought by means of a second-round questionnaire which aimed at acquiring agreement at a level $>50 \%$ but $<75 \%$. This was intended for experts whose views fell between these ranges. This provided them with a chance to possibly change their mind after reviewing the other experts' agreement and justifications. Subsequent Delphi rounds often take the form of structured questionnaires analyzing the response and incorporating feedback of the panel members, which have been found to encourage panel members to become more involved and motivated to participate. ${ }^{32}$ In scoring the outcome of the results, the response alternatives of "agree" and "strongly agree" were grouped together into a positive "agree" response. The data derived from the Likert-scale questions during Phase II were summated to determine each item's score. Summating item scores makes it possible to detect fine discriminations among people with different views. $^{33}$

\section{Ethics and consent to participate}

The study was reviewed and ethical clearance was obtained from the Higher Degrees Committee of the Department of Health Studies at the University of South Africa (UNISA). Ethical approval to conduct the study was obtained from the Addis Ababa Regional Health Bureau ethics committee. Letter of permission to conduct the study was provided from the sub-city administration health departments and the respective health facility officials, and written informed consent was obtained from all respondents prior to collecting the data.

\section{Results}

In Phase I study, of a total of 848 questionnaires administered for exit patient interview, 836 responded, and of all 22 questions administered to health facility manager, 22 responded giving an overall Phase I response rate of 98.6\%. In Phase II, all 20 experts responded to the two rounds of Delphi consensus-seeking questionnaires.

In Phase I patient exist interview respondents, $61.1 \%$ $(n=511)$ were female and $38.9 \%(n=325)$ were male. The majority, $50.6 \%(n=423)$, were $26-45$ years old, while $24.9 \%$ $(n=208)$ were $18-25$ years old and $24.5 \%(n=205)$ were $>46$ years old. Of the respondents, $56.6 \%(n=473)$ had not completed grade $12 ; 27.3 \%(n=228)$ were grade 12 high school graduates or had acquired a higher qualification, while $16.1 \%$ $(n=135)$ had never attended school. Most respondents, $66.3 \%$ $(\mathrm{n}=548)$ had a monthly income $<3,500$ Ethiopian birr (ETB) (equivalent to $\$ 175)$, while only $9.1 \%(n=75)$ had a monthly income $>3,500$ ETB. As many as $24.6 \%(n=203)$ were not able to estimate or did not know their monthly income. Of the respondents attending the health facilities, $43.5 \%(n=362)$ came for the treatment of an acute illness with an onset of $>24$ hours but $<2$ weeks duration, and $12.9 \%(n=107)$ came for the treatment of emergency health problems with an onset of $<24$ hours. This outlined the vast opportunity of informing patients who come for treatment of acute or emergency health conditions of the value and processes of health promotion and disease prevention intervention approaches. Of the respondents, $23.4 \%(n=195)$ sought treatment or follow-up for chronic health conditions with an onset of longer than 2 weeks, and lastly, $11.2 \%(n=93)$ were apparently healthy during the interview time but came to the health facility for some periodic health examination (PHE) or routine check-up. Of the respondents, $91.8 \%(n=766)$ did not have any kind of health insurance or medical coverage.

In Phase I health service manager respondents, 59.1\% $(n=13)$ were male and $40.9 \%(n=9)$ were female, and the majority, $77.3 \%(n=17)$, were under the age of 40 years; $13.6 \%$ $(n=3)$ were aged $40-49$ years; and $9.1 \%(n=2)$ were $>50$ years of age. Most managers, $81.8 \%(n=18)$, had $1-5$ years of managerial experience; $9.1 \%(n=2)$ had 6-10 years' experience, and $9.1 \%(n=2)$ had $>10$ years' experience; The highest educational qualification was either diploma or degree qualified (81.8\%; $\mathrm{n}=18)$ while $18.2 \%(\mathrm{n}=4)$ were medical doctors, of which $13.6 \%(n=3)$ had an additional clinical specialization.

In Phase II, of the 20 experts who were working in Ethiopia, $75 \%(n=15)$ were male while $25 \%(n=5)$ were female. The experts had a minimum work experience of 11 years and a maximum of 35 years (average of 16 years) in the field of health promotion and disease prevention. The majority of experts $(50 \% ; n=10)$ were from hospitals and health centers while $15 \%(n=3)$ working in Ministry of Health and $35 \%(n=7)$ were public health experts working in different non-governmental organizations (NGOs).

\section{Health promotion services provided by health facilities}

Taking into account the selected criteria for the overall measurement of health promotion services rendered as experienced by the patients, of the patients only $2.4 \%(n=20)$ patients received optimal health promotion services relevant to their age and sex from their health service providers. Of all the patients, $37.2 \%(\mathrm{n}=311)$ received some health promotion services but only on a limited number of certain health 
promotion areas, which was considered "sub-optimal," and $60.4 \%(n=505)$ did not receive any of the health promotion services at all. This highlights the extent to which the health care facilities focused on curative health care services without integrating them with the promotive health care aspects (Table 1).

The utilization of health promotion provision approaches varies among health facilities. Asked if the health facilities provided health promotion services to their patients, $90.0 \%(n=20)$ stated that they provided health promotion services to their patients attending the facilities, using different approaches. These health promotion approaches incorporated morning mass education $(100 \% ; \mathrm{n}=20)$; audio/video education materials $(20 \% ; n=4)$; leaflets $(55 \% ; n=11)$; posters $(50 \% ; n=10)$; and one-to-one health promotion services $(55 \% ; n=11)$.

In addition to the underutilization of effective health promotion approaches by health facilities, the availability of patient-specific written materials, particularly for the prevention of chronic diseases and promotion of healthy lifestyles, was lacking at the health facilities. For instance, written materials on health education in the form of a pamphlet, leaflet, or any other form, 90.9\% $(n=20)$ had none on cancer; $86.4 \%(n=19)$ had none on stroke; $77.3 \%(n=17)$ had none on cardiovascular diseases (CVDs); 72.7\% $(n=16)$ had none on hypertension; and $59.1 \%(n=13)$ had none on diabetes. Similarly, written health promotional materials on predisposing factors which may lead to chronic diseases were mostly not available as indicated by the health facility managers; $77.3 \%(n=17)$ had none for alcohol abuse; $72.7 \%$ $(n=16)$ had none for exercise; $68.2 \%(n=15)$ had none for cigarette smoking; and $63.6 \%(n=14)$ had none for nutrition. Of the health service managers, $81.8 \%(n=18)$ described their health promotion service as integrated in the health service provision. However, the integration was more focused on vertical programs, such as tuberculosis (TB), HIV/AIDS, family planning, antenatal care, and expanded immunization, which were mainly supported by the NGOs. Consequently, $68.2 \%(n=15)$ of the health facilities had written promotional material for TB and 59.1\% $(n=13)$ had for HIV/AIDS.

Table I Extent of promotive, preventive, and integrated service provision by the health facilities $(n=836)$

\begin{tabular}{|l|l|l|l|l|l|l|l|l|}
\hline \multirow{2}{*}{ Service type } & \multicolumn{3}{|l|}{ Optimal } & \multicolumn{2}{l|}{$\begin{array}{l}\text { Sub- } \\
\text { optimal }\end{array}$} & \multicolumn{2}{l|}{$\begin{array}{l}\text { Not } \\
\text { provided }\end{array}$} & \multicolumn{2}{l|}{ Total } \\
\cline { 2 - 10 } & N & $\%$ & N & $\%$ & N & $\%$ & N & $\%$ \\
\hline Health promotion & 20 & 2.4 & 311 & 37.2 & 505 & 60.4 & 836 & 100 \\
\hline Disease prevention & 30 & 3.6 & 598 & 71.5 & 208 & 24.9 & 836 & 100 \\
\hline Integrated health service & 7 & 0.8 & 294 & 35.2 & 535 & 64.0 & 836 & 100 \\
\hline
\end{tabular}

\section{Preventive health care}

Of all the patients treated at OPDs and IPDs in the selected health facilities, only $3.6 \%(n=30)$ received optimal disease prevention services relevant to their age and sex; $71.5 \%$ $(\mathrm{n}=598)$ received sub-optimal disease prevention services; and $24.9 \%(n=208)$ received no preventive services. This underscores a very low performance in the integration of preventive health care and the importance of improving preventive services at health facilities (Table 1).

Regarding preventive health services, 59.1\% $(n=13)$ of the health facilities reported that they provided preventive health service by integrating it in the curative health care service while $36.4 \%(n=8)$ provided preventive health care services as a separate unit. This indicated that preventive service provision at health facilities was either integrated with the curative services or provided as a separate entity.

\section{Integrative health care}

IHS provision by health facilities was considered provided to a patient when at least the minimum recommended list of both health promotion and disease prevention services were provided to the patients. Of all patients, only $0.8 \%(n=7)$ received "optimal" integrated health care service, meaning an integrated health promotion and disease prevention service aligned to their sex and age in addition to the curative health care provided by the health facilities. $35.2 \%(n=294)$ received "sub-optimal" integrated promotive, preventive, and curative health care, and $64 \%(n=535)$ did not receive integrated health care. This demonstrates the serious need for the integration of health promotion and disease prevention services with curative health care services (Table 1).

\section{Correlation between promotive, preventive, and integrated health care provision and respondents' biographical, health facility-type, and patient-related characteristics}

As indicated in Table 2, there was a significant but low positive correlation between receiving promotive health care service and respondents who previously received routine check-ups $\left(\mathrm{r}_{\mathrm{s}}=0.194 ; P<0.01\right)$, respondents who were provided with pamphlets or leaflets $\left(\mathrm{r}_{\mathrm{s}}=0.137 ; P<0.01\right)$, respondents' satisfaction with their way of life $\left(\mathrm{r}_{\mathrm{s}}=0.185\right.$; $P<0.01)$, monthly income $\left(\mathrm{r}_{\mathrm{s}}=0.117 ; P<0.01\right)$, and health facility type (hospital vs health center) $\left(\mathrm{r}_{\mathrm{s}}=0.086 ; P<0.05\right)$. There was also a significant but low negative correlation between receiving promotive health care and being worried 
Table 2 Correlation between health promotion, disease prevention, and integrated health care provision and respondents' biographical, health facility, and patient-related characteristics $(n=836)$

\begin{tabular}{|c|c|c|c|c|}
\hline \multicolumn{2}{|l|}{$\begin{array}{l}\text { Biographical, health facility, and } \\
\text { patient-related characteristics }\end{array}$} & \multirow{2}{*}{$\begin{array}{l}\text { Promotion service } \\
\text { provision }\end{array}$} & \multirow{2}{*}{\begin{tabular}{|l|}
$\begin{array}{l}\text { Prevention service } \\
\text { provision }\end{array}$ \\
$0.175^{* *}$ \\
\end{tabular}} & \multirow{2}{*}{$\begin{array}{l}\text { Integrative service } \\
\text { provision }\end{array}$} \\
\hline Facility type (health center vs & Correlation coefficient & & & \\
\hline hospital) & Significance (two-tailed) & .013 & 0.000 & 0.000 \\
\hline & $\mathrm{N}$ & 836 & 836 & 836 \\
\hline \multirow{3}{*}{ Patients have routine check-ups } & Correlation coefficient & $0.194^{* *}$ & $0.189^{* *}$ & $0.259^{* * k}$ \\
\hline & Significance (two-tailed) & 0.000 & 0.000 & 0.000 \\
\hline & $\mathrm{N}$ & 833 & 833 & 833 \\
\hline \multirow[t]{3}{*}{ Satisfaction in ways of life } & Correlation coefficient & $0.185^{* *}$ & $0.152^{* *}$ & $0.151^{\text {w* }}$ \\
\hline & Significance (two-tailed) & 0.000 & 0.000 & 0.000 \\
\hline & $\mathrm{N}$ & 761 & 761 & 761 \\
\hline \multirow[t]{3}{*}{ Patient BMI } & Correlation coefficient & $0.11 I^{a}$ & $0.129^{\mathrm{a}}$ & $0.185^{* *}$ \\
\hline & Significance (two-tailed) & 0.046 & 0.020 & 0.001 \\
\hline & $\mathrm{N}$ & 324 & 324 & 324 \\
\hline \multirow[t]{3}{*}{ Patient age } & Correlation coefficient & -0.010 & $0.209^{* *}$ & $0.157^{* k+}$ \\
\hline & Significance (two-tailed) & 0.771 & 0.000 & 0.000 \\
\hline & $\mathrm{N}$ & 836 & 836 & 836 \\
\hline \multirow{3}{*}{$\begin{array}{l}\text { Written pamphlet or leaflet given to } \\
\text { patients }\end{array}$} & Correlation coefficient & $0.137^{* *}$ & \begin{tabular}{|l|l|}
0.039 \\
\end{tabular} & $0.092^{* * *}$ \\
\hline & Significance (two-tailed) & 0.000 & 0.269 & 0.008 \\
\hline & $\mathrm{N}$ & 822 & 822 & 822 \\
\hline \multirow{3}{*}{ Service site (outpatient or inpatient) } & Correlation coefficient & 0.026 & $0.075^{\mathrm{a}}$ & $0.120^{* * k}$ \\
\hline & Significance (two-tailed) & 0.447 & 0.031 & 0.001 \\
\hline & $\mathrm{N}$ & 836 & 836 & 836 \\
\hline \multirow[t]{3}{*}{ Service type (acute vs chronic) } & Correlation coefficient & $-0.136 * *$ & $0.079^{a}$ & 0.059 \\
\hline & Significance (two-tailed) & 0.000 & $0.04 \mathrm{I}$ & 0.127 \\
\hline & $\mathrm{N}$ & 664 & 664 & 664 \\
\hline \multirow{3}{*}{$\begin{array}{l}\text { Health service bill payment (paid vs } \\
\text { insurance) }\end{array}$} & Correlation coefficient & $-0.073^{\mathrm{a}}$ & $0.104^{* *}$ & $0.122^{* * k}$ \\
\hline & Significance (two-tailed) & 0.036 & 0.003 & 0.000 \\
\hline & $\mathrm{N}$ & 821 & 821 & 821 \\
\hline \multirow[t]{3}{*}{ Monthly income } & Correlation coefficient & $0.117 * *$ & $0.07 I^{\mathrm{a}}$ & 0.031 \\
\hline & Significance (two-tailed) & 0.001 & 0.041 & 0.369 \\
\hline & $\mathrm{N}$ & 826 & 826 & 826 \\
\hline \multirow{3}{*}{$\begin{array}{l}\text { Worried for not having enough } \\
\text { money for rent/mortgage }\end{array}$} & Correlation coefficient & $-0.084^{a}$ & -0.016 & 0.028 \\
\hline & Significance (two-tailed) & 0.015 & 0.646 & 0.414 \\
\hline & $\mathrm{N}$ & 833 & 833 & 833 \\
\hline \multirow{3}{*}{$\begin{array}{l}\text { Worried for not having enough } \\
\text { money for food }\end{array}$} & Correlation coefficient & $-0.185 * *$ & -0.061 & $-0.073^{a}$ \\
\hline & Significance (two-tailed) & 0.000 & 0.081 & 0.035 \\
\hline & $\mathrm{N}$ & 830 & 830 & 830 \\
\hline
\end{tabular}

Notes: ${ }^{* *}$ Correlation is significant at the 0.01 level (two-tailed). ${ }^{2}$ Correlation is significant at the 0.05 level (two-tailed). Abbreviation: BMI, body mass index.

about not having enough money for rent or mortgage $\left(\mathrm{r}_{\mathrm{s}}=-0.084 ; P<0.05\right)$, worried about not having enough money for food $\left(\mathrm{r}_{\mathrm{s}}=-0.282 ; P<0.01\right)$, reason for visit (chronic vs acute; $\mathrm{r}_{\mathrm{s}}=-0.136 ; P<0.01$ ), and health care bill payment $\left(\mathrm{r}_{\mathrm{s}}=-0.073 ; P<0.05\right)$.

There was a significant but low positive correlation between receiving preventive health care service and health facility type (hospital vs health center) $\left(\mathrm{r}_{\mathrm{s}}=0.175 ; P<0.01\right)$, respondents who previously received routine check-up $\left(\mathrm{r}_{\mathrm{s}}\right.$ $=0.189 ; P<0.01)$, respondents' age $\left(\mathrm{r}_{\mathrm{s}}=0.209 ; P<0.01\right)$, patients' body mass index $(\mathrm{BMI})\left(\mathrm{r}_{\mathrm{s}}=0.129 ; P<0.05\right)$, outpatient and inpatient services $\left(\mathrm{r}_{\mathrm{s}}=0.075 ; P<0.05\right)$, satisfaction with their way of life $\left(\mathrm{r}_{\mathrm{s}}=0.129 ; P<0.05\right)$, respondents' reason for visit (acute vs chronic) $\left(\mathrm{r}_{\mathrm{s}}=0.079 ; P<0.01\right)$, and health care bill coverage (pay vs covered through insurance or free) $\left(\mathrm{r}_{\mathrm{s}}=0.104 ; P<0.05\right)$.

There was a significant but low positive correlation between receiving integrated health care service and health facility type (hospital vs health center) $\left(\mathrm{r}_{\mathrm{s}}=0.283 ; P<0.01\right)$, respondents who previously received routine check-ups $\left(\mathrm{r}_{\mathrm{s}}\right.$ $=0.259 ; P<0.01)$, respondents' satisfaction with their way of life $\left(\mathrm{r}_{\mathrm{s}}=0.151 ; P<0.01\right)$, patients' BMI $\left(\mathrm{r}_{\mathrm{s}}=0.158 ; P<0.01\right)$, respondents' age $\left(\mathrm{r}_{\mathrm{s}}=0.157 ; P<0.01\right)$, respondents who were provided with pamphlets or leaflets $\left(r_{s}=0.092 ; P<0.01\right)$, 
outpatient and inpatient services $\left(\mathrm{r}_{\mathrm{s}}=0.120 ; P<0.01\right)$, and health bill coverage $\left(r_{\mathrm{s}}=0.122 ; P<0.01\right)$. There was also a significant but low negative correlation between receiving integrated health care services and being worried about not having enough money for food $\left(\mathrm{r}_{\mathrm{s}}=-0.73 ; P<0.05\right)$.

\section{Reasons for not providing integrated health care}

In this study, triangulating the Phase I and Phase II study findings enabled the researcher to identify the main reasons for not providing an integrated health care service at the health facilities. These included shortage of skilled health staff, equipment, medication, protocols and guidelines, high preventive health care service cost, poor patient awareness, and health professionals' high focus on curative health care.

\section{Shortage of skilled health staff}

In relation to the chronic NCD health service provision, health facilities' readiness in terms of the availability of skilled staff to provide service and counseling was severely compromised. Of the health facilities, only $22.7 \%(n=5)$ had doctors or internists who could diagnose and treat cardiacrelated conditions; $77.3 \%(\mathrm{n}=17)$ had no doctors or internists who could diagnose and treat chronic lung disease conditions; only $13.6 \%(n=3)$ had skilled doctors who could diagnose and treat cerebrovascular incidents; $63.6 \%(n=14)$ did not have doctors who could diagnose and treat patients with chronic liver diseases; $50 \%(n=11)$ did not have staff who could diagnose and treat diabetic patients; only $9.1 \%(n=2)$ had doctors, internists, or oncologists who could diagnose and treat cancer patients; $59.1 \%(n=13)$ did not have staff who could provide health education and counseling on the prevention of cancer; and $50 \%(\mathrm{n}=11)$ did not have staff who could diagnose and treat diseases related to nutrition.

The experts agreed $(90 \% ; n=18)$ the shortage of health education and promotion specialists in the country to coordinate, plan, and implement client-centered learning. Of the health facilities, $63.6 \%(n=14)$ did not have trained staff who could provide education to patients on how to promote a healthy lifestyle and prevent diabetes, and 50\% $(n=11)$ did not have trained staff to promote how to prevent cigarette smoking. Of the health facilities, $22.7 \%(n=5)$ had trained staff who could provide rehabilitation for patients in need of ceasing smoking; $9.1 \%(n=2)$ had trained health staff who provided rehabilitation for patients who needed to cease alcohol addiction; $63.6 \%(n=14)$ did not have trained staff who could provide health education on the importance of physical exercise and living a healthy lifestyle; and 9.1\% $(n=2)$ had a physiotherapist or trained health staff to provide service and advice on physical exercise. The experts $(80 \% ; n=16)$ also agreed that the majority of health service providers lacked the knowledge or skills for the prevention and treatment of hyperlipidemia (elevated blood cholesterol level), such as providing advice on lifestyle, healthy diet, physical exercise, and treating patients suffering from the disease. The experts also pointed out that health professionals' knowledge in providing client-centered support on cancer screening, diagnosis, and treatment needs improvement. The experts $(95 \% ; n=19)$ recommended that preventive medicine should be specifically incorporated in health professionals' training by revising the curriculum of medical students. Health service providers' competencies (knowledge, skills, and attitudes) regarding promotive and preventive health care should be developed and enhanced by means of well-planned and regular in-service training.

However, the existence of sufficient skilled professionals does not mean the provision of the desired integrated health care provision. For instance, the findings from Phase 1 indicated that of the health facilities, $100 \%(n=22)$ had sufficient skilled staff who could measure BP, but only $47.2 \%(n=394)$ of the patients' BP was measured. Similarly, of the health facilities, 77.3\% $(n=17)$ had staff who could provide health education on raised blood cholesterol level, but $81.1 \%(n=678)$ of patients indicated that their blood cholesterol levels had never been checked and $81.8 \%(n=18)$ of health facilities did not have enough health education or promotion resource materials (for raised blood cholesterol levels) for their patients.

\section{Shortage of medical equipment}

The assessment conducted at health facilities for the availability of equipment needed to diagnose or treat NCDs showed substantial shortages. About $68 \%(n=15)$ of the health facilities did not have equipment to measure blood cholesterol levels; none $(0 \% ; n=0)$ had the necessary equipment to diagnose coronary heart disease; and $13.6 \%(n=3)$ had only EKG equipment. For chronic lung disease diagnosis, only $13.6 \%(n=3)$ of the health facilities had X-ray machines while $0 \%(n=0)$ had a spirometer to conduct lung function tests. Of the health facilities, $0 \%(\mathrm{n}=0)$ had computerized tomography scans or Magnetic Resonance Imaging equipment for diagnosis and investigation of the extent of stroke damage to the brain; 36.4\% $(n=8)$ had laboratory tests for liver function; only $13.6 \%(n=3)$ had ultrasound equipment; $31.8 \%(n=7)$ did not have laboratory test for identifying hepatitis virus; and $18.2 \%(n=4)$ did not have a simple laboratory test for fasting blood sugar. 
The investigation for early diagnosis of cancer was mainly deficient in most of the health facilities. Of the health facilities, $54.5 \%(n=12)$ did not conduct blood laboratory tests for blood count and morphology for diagnosis of blood cancer, such as leukemia; only $4.5 \%(n=1)$ conducted fine needle aspiration tests and 9.1\% $(n=2)$ conducted sigmoidoscopic or endoscopic examinations for the diagnosis of early malignant change of tissues or cancer; $0 \%(n=0)$ performed tissue biopsy tests for distinguishing cancerous growths from non-cancerous lesions, mammographic examination for early diagnosis of breast cancer, or prostate antigen tests for the diagnosis of prostatic cancer.

The rehabilitative preparedness of health facilities for diseases related to addiction or physical well-being was also grossly deficient. Of the health facilities, $0 \%(n=0)$ had an addiction rehabilitation unit or could conduct laboratory tests to monitor blood nicotine levels for patients who smoked cigarette and wanted to quit smoking, and $0 \%(\mathrm{n}=0)$ had laboratory facilities to monitor blood alcohol levels and a rehabilitation unit to help quit alcohol abuse. Of the health facilities, only $4.5 \%(n=1)$ had equipment for physical rehabilitation or physical exercise for patients who required physiotherapy or exercise. Of the experts, $90 \%(n=18)$ suggested that the government should allocate/budget for medical equipment and import the technologies and medical supplies essential for health promotion and disease prevention services. The experts agreed $(85 \% ; n=17)$ that it was expensive and not feasible to have physical exercise and rehabilitation units in all health facilities, but all hospitals should be equipped with basic exercise devices for rehabilitation purposes and physical exercise sites, gyms, and physiotherapy units be made available at affordable prices for the general population.

\section{Unavailability of medication}

The assessment of the availability of specific chronic disease medications at the health facilities revealed major shortages. Of the health facilities, 9.1\% $(\mathrm{n}=2)$ did not have adequate antihypertensive drugs to treat their patients; only $31.8 \%(n=7)$ had the proper medication to treat CVDs; only $27.3 \%(n=6)$ had medication to treat diabetes; $68.2 \%(n=15)$ lacked enough medication for decreasing elevated cholesterol levels; $72.7 \%$ $(n=16)$ did not have drugs for the prevention and treatment of stroke; and $50 \%(\mathrm{n}=11)$ did not have medications to treat chronic lung disease conditions. The shortage of medication also extended to diseases related to nutrition and cancer. Of the health facilities, $36.4 \%(n=8)$ did not have nutrient formula to treat patients with nutritional deficiencies such as kwashiorkor and marasmus; only $4.5 \%(n=1)$ of facilities could provide chemotherapy for patients suffering from cancer; and $0 \%(n=0)$ were able to provide radiotherapy for cancer patients.

\section{Lack of protocols and guidelines}

In order to integrate promotive and preventive health care into curative health care, protocols and guidelines for specific types of diseases, such as hypertension, hyperlipidemia, and diabetes, were found lacking. Of the experts, 95\% $(n=19)$ agreed that health facilities need to prepare reference handbooks, job aids, and promotive and preventive service checkup protocols for the health workers. These could be combined in patient care guidelines for the specific conditions.

\section{Barriers related to health service cost}

The living standard of the population who seeks public health care is at a low level; the monthly salary of most $(73.3 \%$; $\mathrm{n}=606$ ) of the patients who attended the health facilities was $<5,000$ ETB (equivalent to 250 USD). Most health facilities included waived or free of charge services to patient treatment services except one $(4.5 \% ; n=1)$ health facility that made all the patients pay to receive curative health care. Of the patients, $84.5 \%(n=703)$ paid for the service they received while $12.7 \%(\mathrm{n}=106)$ received a free service. Moreover, only $1.4 \%(n=12)$ of the patients were covered by insurance for the treatment they received from the health facilities. This was mainly because of the health facilities, 95\% $(n=19)$ had not initiated a structured insurance coverage for promotive and preventive services while $36.3 \%(n=8)$ facilities claimed that they provided selected promotive and preventive services for free. Of the patients, $62.7 \%(n=520)$ were unable to pay for an annual medical check-up that costs 1,000-2,000 ETB (\$50-\$100). Most of the patients, therefore, could not afford annual check-ups for preventive service, which indicated that the economic inaccessibility of preventive health care can be considered one of the reasons they did not use the services. Of the health facilities, $63.6 \%(n=14)$ did not provide preventive health care services, such as PHE, and 50\% $(n=11)$ did not provide case finding or integrate the payment modalities in the insurance system. Of the experts, $90 \%(n=18)$ recommended that preventive services such as periodic health check-ups should be covered through the community or individual insurance system and that patients who have positive results upon screening and who cannot afford the treatment should be supported by the health insurance system for their specific health problem. 


\section{Low patient health literacy}

Health literacy involves the integration of a wide variety of individual skills, communication skills of health care professional, health care practices, and system processes. ${ }^{34}$ Health literacy is important because it can improve patients' promotive and preventive health care use, which has an impact on their social and economic well-being. The experts $(95 \% ; n=19)$ agreed that enhancing patients' awareness of the importance of available health promotion and disease prevention services is necessary to make each patient ask for critical preventive services whenever this was overlooked by health service providers. For such purposes, the experts recommended that health facilities prepare promotive and preventive health care health education materials, distribute leaflets on specific diseases, and use the media to increase the population's awareness of NCDs. The experts $(95 \% ; n=19)$ agreed further that health facilities must regularly inform patients and individuals visiting the health facilities of the importance of preventive health care, such as measuring BP, to increase the demand for such services and benefit from the service by linking it with the health care insurance system. Creating a population that can demand comprehensive and integrated health care from the health facilities is essential instead of just receiving curative care for the presenting sickness.

\section{Health professionals focus on curative health care}

Of the patients, $64 \%(n=535)$ did not receive any health promotion or disease prevention service in addition to the curative health care they received. This indicated that the health professionals' focus was mainly on curative health care. During the consensus-seeking process, the experts $(95 \% ; n=19)$ agreed that the perception of NCDs (including hypertension) and their risk factors being a disease of the affluent is still predominant among health workers. The experts advised that health professionals should routinely and strictly link the health promotion and disease prevention services (such as advice on healthy diet and physical exercise) to curative health care services.

\section{Discussion}

When assessed on the provision of health promotion services, majority of patients (60.4\%) did not receive any health promotion services at all (Figure 1). There was a positive association for receiving promotive health care services if patients had had previous routine health check-ups, health facilities provided patients with pamphlets or leaflets, patients were satisfied with their way of life, patients' monthly income increased, and if treatment was provided at hospitals rather than health centers. Health facilities can work on factors positively associated with receiving health promotion services to improve the quality of patient care. This indicates respondents who had periodic health check-ups tended to receive more promotive health care during their other visits to health facilities, indicating an added value of having periodic health check-ups. Health facilities' readiness in terms of providing written health promotive materials to patients improved the

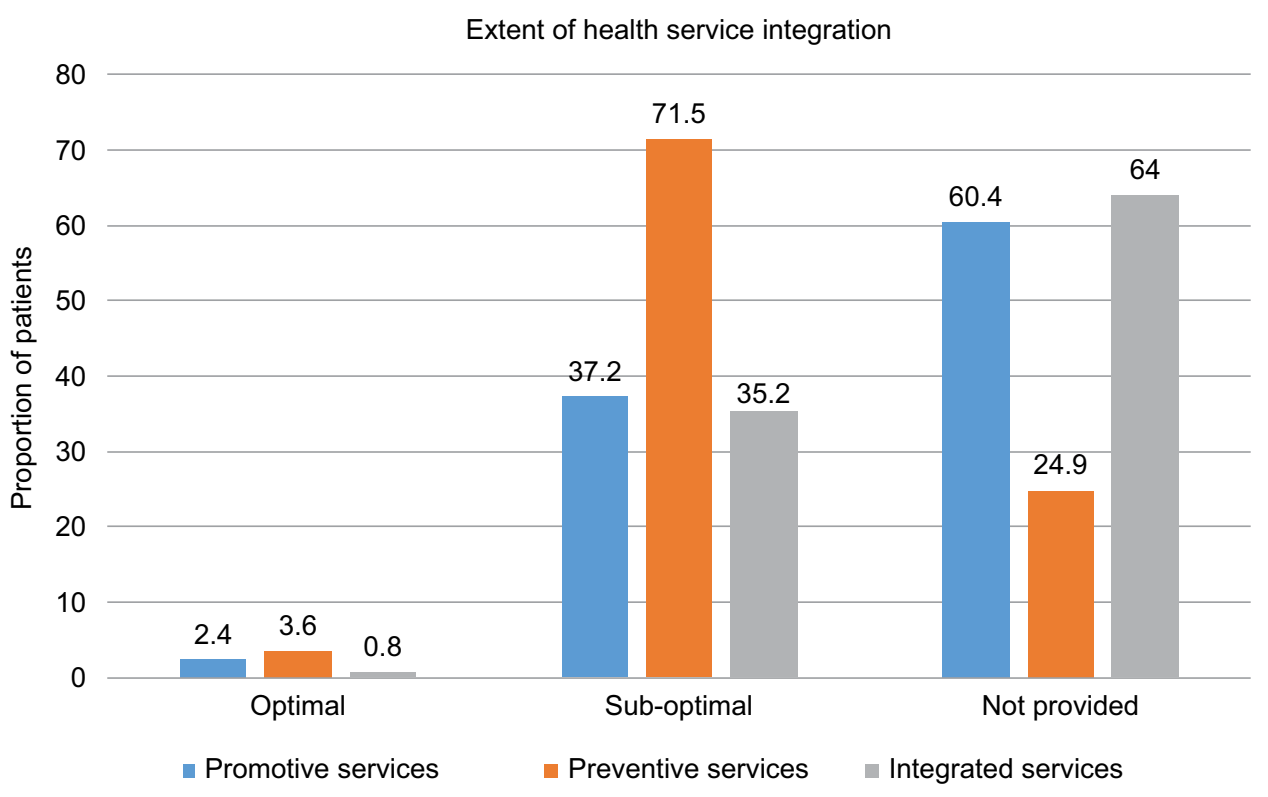

Figure I Patients received integrated health care services. 
integration of promotive health care to the routine health care. Respondents who self-reported on being satisfied with their way of life received more health promotive services, thus indicating the role of their psycho-social condition in receiving health promotion services at health facilities. Health centers can start providing health promotion services as hospitals do, since most of the promotive services do not require additional equipment.

Less promotive health care was received by patients who reported being worried about not having enough money for the rent or mortgage or for food, by patients who paid their health care bill, and by patients with chronic health problems. This indicates respondents who reported being worried over not having enough money for rent or mortgage or for food tended to receive lesser promotive health care services, respondents who paid the health care bill received less promotive health care than those who had insurance coverage or received free services, and respondents who visited with chronic problems received less promotive health care than those who sought treatment for acute diseases. This could result when patients do not have financial security for the health care they need; they tend not to seek the service and chronic patients are being provided with curative-focused services overlooking their need of promotive aspects. These correlations also point to the importance of health professionals investigating the social determinants of patients' health, such as stress factors related to housing and food, and aligning the provision of health promotion services at health facilities. Patients who pay for the treatment of specific illnesses need to be provided with the health promotion services as those who receive treatment through insurance or receive free services. In addition, health professionals' focus on health promotion for patients seeking treatment for chronic illnesses needs to be improved when compared to acute patients.

When assessed on the provision of disease prevention services, of all the patients treated at OPDs and IPDs at the selected health facilities, only few (3.6\%) received optimal disease prevention services, indicating that the opportunity for disease prevention was grossly missed. Respondents received more preventive health care at hospitals than health centers, respondents who were discharged from inpatient service received more preventive health care service than the outpatients, as their age increased respondents received more preventive health care from the health facilities (both hospitals and health centers), respondents who paid received less preventive health care than respondents who had insurance or free service. This finding corresponds to the global truth; even in well-developed countries, the levels of chronic disease control, such as for hypertension, are particularly low for people who are uninsured or do not have source of health care. ${ }^{35}$ Patient with chronic diseases received more preventive services than acute diseases, respondents who had routine periodic check-ups received more preventive services during their health care visits, possibly because they were viewed and consulted at the health care facility. Respondents who self-reported being satisfied with their life tended to receive more preventive services at health facilities, which supported Dolan et al's finding that higher life satisfaction is associated with higher use of several preventive services. ${ }^{36}$ All factors positively associated with the provision of preventive health care can possibly be dealt with by the health centers and hospitals. The health centers can improve the provision of preventive health care by integrating curative health care and by availing preventive health care linking with insurance system or as part of the waived or free service package. The finding highlighted that the preventive health care service in OPDs needs improvement and tapering in such a way that patients of all age groups benefit from preventive health care. Having routine periodic health check-ups is important to receive further preventive health care services while patients visit health facilities for other reasons. Assessing patients' psycho-social aspects can help in addressing the preventive health care needs of patients who are not satisfied with their way of life.

When viewed holistically, of all the patients, only few $(0.8 \%)$ had received an integrated health promotion and disease prevention service while majority $(64 \%)$ did not receive integrated health care services at all (Figure 1). This demonstrates the grave need for health promotion and disease prevention service integration in existing infrastructure for curative health care services. ${ }^{37}$ Integrated health care services were provided at hospitals than health centers to respondents who had routine check-ups than to those who had not, to respondents who were satisfied with their way of life than those who were not, and to respondents who had increased BMI. The provision of integrated health care in health facilities also increased as respondents' age increased, especially for respondents to whom written materials were provided, at IPDs than at OPDs, and for respondents who had insurance health coverage or received free service than those who paid. The actions to improve the provision of integrated services are linked to those required to improve the health promotion and disease prevention services in the same way that the provision of integrated services depends on the provision of health promotion and disease prevention services. Therefore, it is feasible to address the factors asso- 
ciated with the provision of integrated services by tackling the factors associated with the provision of health promotion and disease prevention health care services. Actions that target the study findings on delivering an integrated health care service at health facilities can improve the service. For example, integrated health care service was not provided to all age groups, including young patients; health centers and hospitals did not provide structured integrated health care services to all their patients both at OPDs and IPDs; and not all patients, irrespective of their bill payment coverage, were able to get access to the service. The finding also highlights the importance of encouraging periodic health check-ups and assessing patients' BMI routinely; providing written materials prepared for patients' specific demands; and assessing patients' psycho-social factors affecting their satisfaction with life and their health.

Considering the findings from the study, on barriers of optimal integration of promotive and preventive health care in curative health services, a comprehensive approach is recommended. Health facility readiness (availing qualified staffs, equipment, and supplies) for integrated health care provision is critical. Medical school curriculum should be re-designed to include promotive and preventive medicines in all categories of health professionals such as MDs, nurses, nutritionists, and physiotherapists. Health service providers should receive comprehensive ongoing in-service training programs focusing on promotive and preventive health care. The training needs to be aimed at enabling a paradigm shift from focusing only on patient complaint treatment to comprehensive patient-centered integrated health care approach, improving health professionals' skill and knowledge on the importance of PHE and case finding, and in using patients' presence at a health facility as an opportunity to provide preventive health recommended for the patients' age, gender, and disease condition. Health service providers should align patient age, reason for clinic visit, and satisfaction to their way of life with their need of specific integrated health care services. Integrated health care needs to be directed by means of patient care guidelines at health facilities and selected health promotion, disease prevention, and treatment interventions should be included in the health insurance system. Written promotional materials for common chronic diseases should be standardized and available at all health facilities. This is because the differences in health literacy and poor health are consistently associated with minimal use of preventive services and poorer overall health status and higher risk of mortality. ${ }^{34}$ To ensure standardized integrated health care provision, quality checks on the type of integrated health care provided have to be frequently conducted. This includes conducting regular clinical and record audits for service improvement.

\section{Conclusion}

Health service providers were not routinely conducting patient-specific health promotion, disease prevention, and integrated health care services along with the curative services they provided. As the result of poor integration of health service provision, the use of opportunities of patients' presence at health facilities for health promotion and disease prevention purpose was underutilized. The factors related for not providing integrated services were shortage of skilled health staff, medical equipment and medication, lack of protocols and guidelines, preventive health service cost, patient health literacy, and health professionals' focus on curative health care. This entails the need for addressing the barriers in order to optimally integrate health promotion and preventative health care services to the routine curative health care services of health facilities.

\section{Supporting data}

Supporting data can be accessed on request to the authors.

\section{Acknowledgments}

Our sincere gratitude is to the University of South Africa, for permission to conduct the study and for the Higher Degrees Committee of the Department of Health Studies, University of South Africa (UNISA), for ethical clearance and permission. We are grateful to the Addis Ababa Regional Health Bureau, sub-city administration health departments and the respective health facilities, for providing permission to conduct the study in their facilities. The study would have not been possible had it not been for the patients, the health facility managers, and health professionals, generously sharing their expertise, patience, and time. We are thankful to Rina Coetzer, for her patient and professional finalizing of the manuscript, and to Iauma Cooper, for critically and professionally editing the language and content of the manuscript. The study was not funded by any grant.

\section{Author contributions}

Both authors contributed toward data analysis, drafting and critically revising the paper, gave final approval of the version to be published, and agree to be accountable for all aspects of the work. NFW, designed the study concept note, developed proposal and thesis, and conducted data collection analysis, interpretation and write-up. MCB closely advised 
and supported NFW in the development of study concept note, developing the proposal and thesis, and data analysis, interpretation and write-up.

\section{Disclosure}

The authors report no conflicts of interest in this work.

\section{References}

1. Bonita R, Beaglehole R, Kjellstrom T. Basic Epidemiology. 2nd ed. Vol. 103. Geneva: WHO; 2006.

2. Tengland P-A. Health promotion or disease prevention: a real difference for public health practice? Health Care Anal. 2010;18(3):203-221.

3. Institute for Clinical Systems Improvement (ICSI). Health Care Guideline: Healthy Lifestyles. 4th ed. Bloomington, MN: ICSI; 2011:9.

4. Ballweg R, Sullivan EM, Brown D, Vetrosky D. Physician Assistant: A Guide to Clinical Practice. 4th ed. New York: Elsevier; 2008:278.

5. World Health Organization (WHO). Health Promotion: THE OTTAWA CHARTER for Health Promotion. Geneva: WHO; 2012. http://www. who.int/healthpromotion/conferences/previous/ottawa/en/index.html. Accessed August 25, 2012.

6. International Epidemiological Association. Dictionary of Epidemiology. 5th ed. Vol. 192. Porta M, editor. New York: Oxford University Press; 2008.

7. Katz DL, Ali A. Preventive Medicine, Integrative Medicine and the Health of the Public. New York: WHO; 2009:3-4.

8. Donev D, Pavlekovic G, Kragelj LZ. Health Promotion and Disease Prevention: A Handbookfor Teachers, Researchers, Health Professionals and Decision Makers. London: Institute of Social Medicine; 2007:17-24.

9. Institute for Clinical Systems Improvement (ICSI). Health Care Guideline: Preventive Services for Adults. 17th ed. Bloomington, MN: ICSI; 2011:8-11.

10. Shires DA, Stange KC, Divine G, et al. Prioritization of evidence-based preventive health services during periodic health examinations. $A m J$ Prev Med. 2012;42(2):164-173.

11. World Health Organization (WHO). Political Declaration of the UN High-Level Meeting on the Prevention and Control of Non-Communicable Diseases (NCDS): Key Points; 2011. Available from: http://www.who.int/ nmh/events/un_ncd_summit2011/en/. Accessed September 26, 2014.

12. Beaglehole R, Bonita R, Horton R, et al. Priority actions for the noncommunicable disease crisis. Lancet. 2011;377(9775):1438-1447.

13. World Health Organization (WHO). Preventing Chronic Diseases: A Vital Investment. WHO Global Report. Geneva: WHO; 2005:3.

14. World Health Organization (WHO). Global Status Report on NonCommunicable Diseases, 2010. Geneva: WHO; 2011:9-16.

15. World Health Organization (WHO). Non-Communicable Diseases Country Profiles, 2011. Geneva: WHO; 2011:73.

16. Ethiopia Ministry of Health. Health Sector Development Program III Annual Performance Report. EFY 2002 (2009/10). Addis Ababa: Government Printer; 2010:77.

17. Misganawa, Mariam DH, Araya T, Ayele K. Epidemiology of major non-communicable diseases in Ethiopia: a systematic review. $J$ Health Popul Nutr. 2014;32(1):1-13.
18. Basker GV. A review on hazards of khat chewing. Int J Pharm Pharm Sci. 2013;5(3):74-77.

19. Tesfaye F, Byass P, Wall S. Population based prevalence of high blood pressure among adults in Addis Ababa: uncovering a silent epidemic. BMC Cardiovasc Disord. 2009;9:39.

20. Ethiopia Ministry of Health. National Health Account (NHA V): Household Health Service Utilization and Expenditure Survey EFY 2003 (2010/11). Addis Ababa: Government Printer; 2014:51-78.

21. Global Health Action (GHA). Adult Non-Communicable Disease Mortality in Africa and Asia: Evidence from In-Depth Health and Demographic Surveillance System Sites. Atlanta, GA: GHA; 2014. Available from: http://www.globalhealthaction.net/index.php/gha/ article/. Accessed September 26, 2014.

22. Bygbjerg IC. Double burden of non-communicable and infectious diseases in developing countries. Science. 2012;337(6101): $1499-1501$.

23. Wolff LS, Massett HA, Weber D, Mockenhaupt RE, Hassmiller S, Maibach EW. Opportunities and barriers to disease prevention counseling in the primary care setting: a multisite qualitative study with us health consumers. Health Promot Int. 2010;25(3):265-276.

24. World Health Organization (WHO). Prevention and Control of NonCommunicable Diseases: Guidelines for Primary Health Care in LowResource Settings. Malta: WHO; 2012:34.

25. Wilkinson J, Bass C, Diem S. Preventive Services for Adults. Bloomington, MN: ICSI; 2013.

26. Ethiopia Ministry of Health Policy and Planning Directorate. Health and Health-Related Indicators. Addis Ababa: Government Printer; 2011:54-62.

27. Turner AG. Sampling Strategies. New York: UN Secretariat Statistics; 2003:12.

28. Fox N, Hunn A, Mathers N. Sampling and Sample Size Calculation. Vol. 18. Nottingham: The NIHR RDS for the East Midlands; 2007.

29. Centres for Disease Control and Prevention. Behavioral Risk Factor Surveillance System Questionnaire (BRFSS). Atlanta, GA: CDC; 2011. Available from: http://www.cdc.gov/brfss/. Accessed August 25, 2012.

30. Sherri LJ. Research Methods and Statistics: A Critical Thinking Approach. 3rd ed. Belmont: Wadsworth, Cengage Learning; 2009:115.

31. Chia-Chien H, Braina. The Delphi technique: making sense of consensus. Practical assessment. Research and Evaluation. 2007;12:10.

32. Keeney S, Hasson F, McKenna H. The Delphi Technique in Nursing and Health Research. Vol. 46. Chichester: Wiley-Blackwell; 2011.

33. Polit DF, Beck CT. Nursing Research: Principles and Methods. 8th ed. Vol. 297. Philadelphia: Lippincott Williams \& Wilkins; 2008.

34. Registered Nurses' Association of Ontario. Facilitating Client Centered Learning. Best Practice Guideline. Toronto: Registered Nurses' Association of Ontario; 2012:14-17.

35. Centres for Disease Control and Prevention. Use of selected clinical Preventive services among adults — United States, 2007-2010. MMWR Morb Mortal Wkly Rep. 2012;61-99.

36. Dolan P, Kavetsos G, Tsuchiya A. Sick but satisfied: the impact of life and health satisfaction on choice between health scenarios. $J$ Health Econ. 2013;32(4):708-714.

37. Ethiopia Ministry of Health. Health Sector Transformation Plan 2015/2016-2019/2020. Addis Ababa: Government Printer; 2015;99.
Journal of Multidisciplinary Healthcare

\section{Publish your work in this journal}

The Journal of Multidisciplinary Healthcare is an international, peerreviewed open-access journal that aims to represent and publish research in healthcare areas delivered by practitioners of different disciplines. This includes studies and reviews conducted by multidisciplinary teams as well as research which evaluates the results or conduct of such teams or health
Dovepress

care processes in general. The journal covers a very wide range of areas and welcomes submissions from practitioners at all levels, from all over the world. The manuscript management system is completely online and includes a very quick and fair peer-review system. Visit http://www.dovepress.com/ testimonials.php to read real quotes from published authors. 